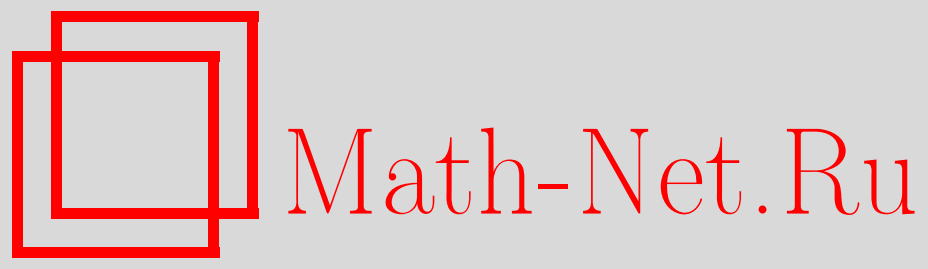

А. А. Комеч, А. И. Комеч, Вариант теоремы Титчмарша о свертке для распределений на окружности, Функи. анализ и его прил., 2013, том 47, выпуск 1, 26-32

DOI: https://doi.org/10.4213/faa3102

Использование Общероссийского математического портала MathNet.Ru подразумевает, что вы прочитали и согласны с пользовательским соглашением http://www . mathnet.ru/rus/agreement

Параметры загрузки:

IP : 3.85 .73 .92

26 апреля 2023 г., $17: 50: 07$

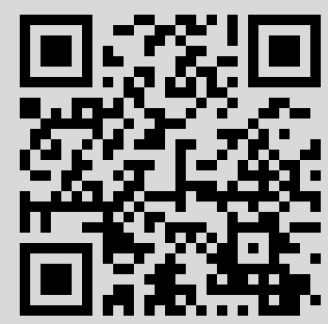


Функционалъный анализ и его приложения

2013, т. 47, вып. 1, с. 26-32

УДК 517.71

\title{
Вариант теоремы Титчмарша о свертке для распределений на окружности
}

\author{
(C) 2013. А. А. Комеч, А. И. Комеч
}

\begin{abstract}
Доказана версия теоремы Титчмарша для распределений на окружности. Мы показываем, что некоторая «наивная форма» теоремы Титчмарша может нарушаться, однако такое нарушение имеет место только в случае свертки распределений, которые обладают определенными свойствами симметрии.
\end{abstract}

1. Введение. Теорема Титчмарша о свертке [6] гласит, что для любых двух распределений с компактным носителем $f, g \in \mathscr{E}^{\prime}(\mathbb{R})$ имеет место соотношение

$\inf \operatorname{supp} f * g=\inf \operatorname{supp} f+\inf \operatorname{supp} g, \quad \sup \operatorname{supp} f * g=\sup \operatorname{supp} f+\sup \operatorname{supp} g$.

Лионс [4] сформулировал ее многомерный аналог, доказав, что для распределений $f, g \in \mathscr{E}^{\prime}\left(\mathbb{R}^{n}\right)$ выпуклая оболочка носителя распределения $f * g$ равна сумме выпуклых оболочек носителей распределений $f$ и $g$. Различные доказательства теоремы Титчмарша содержатся в [7, гл. VI] (в стиле вещественного анализа), в [1, теорема 4.3.3] (à la гармонический анализ), а также в [3, лекция 16] (подход, основанный на комплексном анализе).

В этой работе мы обобщим теорему Титчмарша на случай периодических распределений, которые мы рассматриваем как распределения на окружности, или, точнее, на торе $\mathbb{T}:=\mathbb{R} / 2 \pi \mathbb{Z}$.

Отметим, что в алгебре распределений на окружности относительно операции свертки есть делители нуля. В самом деле, для любых двух распределений $f, g \in \mathscr{E}^{\prime}(\mathbb{T})$ имеем

$$
\left(f+S_{\pi} f\right) *\left(g-S_{\pi} g\right)=f * g+S_{\pi}(f * g)-S_{\pi}(f * g)-f * g=0 .
$$

Здесь $S_{y}, y \in \mathbb{T}$, является оператором сдвига, который определен на $\mathscr{E}(\mathbb{T})$ посредством равенства

$$
\left(S_{y} f\right)(\omega)=f(\omega-y),
$$

которое понимается в смысле распределений. Тем не менее случаи, когда теорема Титчмарша «не выполняется» в некоторой наивной форме, могут быть явно охарактеризованы, приводя к версии теоремы Титчмарша для распределений на окружности (теорема 1).

Наш интерес к свойствам свертки на окружности обусловлен приложениями к теории аттракторов для конечных разностных схем, возникающих при моделировании нелинейных дисперсионных уравнений. В работе [2] мы рассмотрели слабый аттрактор решений конечной энергии $\mathbf{U}(1)$-инвариантного уравнения Клейна-Гордона в одном пространственном измерении, взаимодействующего с нелинейным осциллятором. Мы доказали, что аттрактор всех уравнений конечной энергии образован множеством всех солитоноподобных решений вида 
$\phi_{\omega}(x) e^{-i \omega t}$, где $\omega \in \mathbb{R}$ и $\phi_{\omega} \in H^{1}(\mathbb{R})$. Общая стратегия доказательства заключается в рассмотрении омега-предельных траекторий решения конечной энергии $\psi(x, t) \in \mathbb{C}$. Омега-предельные траектории определяются как решения с данными Коши в омега-предельных точках множества $\{(\psi(t), \dot{\psi}(t)): t \geqslant 0\}$, где предел берется в полунормах энергетического пространства. Для доказательства сходимости к множеству солитонных решений мы доказывали, что спектр каждой омега-предельной траектории заключен внутри спектральной щели. Применяя после этого теорему Титчмарша о свертке к уравнению, которому удовлетворяет омега-предельная траектория, можно заключить, что ее временной спектр состоит из не более чем одной частоты, и, таким образом, любая омега-предельная траектория является уединенной волной (либо нулевым решением). Мы ожидаем, что такой подход можно будет обобщить на случай уравнения Клейна-Гордона на дискретном пространстве-времени [5]. Основное отличие заключается в том, что теперь область частот представлена окружностью (а не вещественной осью), а в существенном спектре имеется не одна, а две щели. Таким образом, для анализа спектра омега-предельной траектории требуется версия теоремы Титчмарша для распределений с носителем на двух интервалах окружности.

Благодарность. Авторы глубоко признательны Е. А. Горину за интерес и поддержку.

2. Основные результаты. Для $I \subset \mathbb{T}$ и $n \in \mathbb{N}$ положим

$$
\mathscr{R}_{n}(I)=\bigcup_{k \in \mathbb{Z}_{n}} S_{2 \pi k / n} I, \quad \text { где } \mathbb{Z}_{n}=\mathbb{Z} \bmod n .
$$

Пусть $f, g \in \mathscr{E}^{\prime}(\mathbb{T})$. Пусть $I, J \subset \mathbb{T}$ - два замкнутых интервала, таких, что supp $f \subset \mathscr{R}_{n}(I)$, supp $g \subset \mathscr{R}_{n}(J)$, и предположим, что не существует замкнутого интервала $I^{\prime} \subsetneq I$, такого, что supp $f \subset \mathscr{R}_{n}\left(I^{\prime}\right)$, а также не существует замкнутого интервала $J^{\prime} \subsetneq J$, такого, что supp $g \subset \mathscr{R}_{n}\left(J^{\prime}\right)$.

Замечание 1. Для $f, g \in \mathscr{E}^{\prime}(\mathbb{T})$ интервалы $I$ и $J$ играют роль, аналогичную «выпуклым оболочкам» носителей.

Теорема 1 (теорема Титчмарша для распределений на окружности). Пусть $n \in \mathbb{N}, n \geqslant 2$. Предположим, что

$$
|I|+|J|<2 \pi / n
$$

Пусть $K \subset I+J \subset \mathbb{T}-$ замкнутый интервал, такой, что supp $f * g \subset \mathscr{R}_{n}(K)$. Если $\lambda:=\inf K-\inf I-\inf J>0$, то найдутся $\alpha, \beta \in \mathbb{C}, \alpha^{n}=\beta^{n}=1, \alpha \neq \beta$, maкuе, чmо

$$
\left.\begin{array}{rl}
\left.\left(\sum_{k \in \mathbb{Z}_{n}} \alpha^{k} S_{2 \pi k / n} f\right)\right|_{(\sup I-2 \pi / n, \inf I+\lambda)} & =0, \\
\left.\inf \operatorname{supp}\left(\sum_{k \in \mathbb{Z}_{n}} \alpha^{k} S_{2 \pi k / n} g\right)\right|_{(\sup J-2 \pi / n, \inf J+\lambda)} & =\inf J,
\end{array}\right\}
$$


Замечание 2. Соотношения (6) получаются из (5) благодаря симметричной роли распределений $f$ и $g$. Соотношение $\alpha \neq \beta$ следует из сравнения (5) и (6). В самом деле, первое равенство в (5) приводит к неравенству

$$
\left.\inf \operatorname{supp}\left(\sum_{k \in \mathbb{Z}_{n}} \alpha^{k} S_{2 \pi k / n} f\right)\right|_{I} \geqslant \inf I+\lambda>\inf I,
$$

что противоречило бы первому соотношению в (6) в случае, если бы мы имели $\alpha=\beta$.

Применяя отражение к $\mathbb{T}$, мы также получаем такой результат:

Следствие 1. Если $\rho:=\sup I+\sup J-\sup K>0$, то найдутся $\alpha, \beta \in \mathbb{C}$, $\alpha^{n}=\beta^{n}=1, \alpha \neq \beta$, такие, что

$$
\begin{aligned}
& \left.\begin{array}{l}
\left.\left(\sum_{k \in \mathbb{Z}_{n}} \alpha^{k} S_{2 \pi k / n} f\right)\right|_{(\sup I-\rho, \inf I+2 \pi / n)}=0, \\
\left.\left(\sum_{k \in \mathbb{Z}_{n}} \alpha^{k} S_{2 \pi k / n} g\right)\right|_{(\sup J-\rho, \inf J+2 \pi / n)}=\sup J,
\end{array}\right\} \\
& \left.\begin{array}{rl}
\sup \operatorname{supp} & \left.\left(\sum_{k \in \mathbb{Z}_{n}} \beta^{k} S_{2 \pi k / n} f\right)\right|_{(\sup I-\rho, \inf I+2 \pi / n)} \\
& =\sup I, \\
\left.\left(\sum_{k \in \mathbb{Z}_{n}} \beta^{k} S_{2 \pi k / n} g\right)\right|_{(\sup J-\rho, \inf J+2 \pi / n)} & =0 .
\end{array}\right\}
\end{aligned}
$$

Таким образом, если $K \subsetneq I+J$ (грубо говоря, некоторая наивная форма теоремы Титчмарша о свертке не выполнена), то и $f$, и $g$ обладают некоторыми свойствами симметрии на $\mathscr{R}_{n}(U)$ и на $\mathscr{R}_{n}(V)$, где открытые непересекающиеся интервалы $U$ и $V$ могут быть выбраны так, что $U \cup K \cup V \supset I+J$.

В случае $n=2$ получаем такой результат:

Следствие 2. Пусть $n=2, f, g \in \mathscr{E}^{\prime}(\mathbb{T})$, и пусть $I, J, K$ такие же, как в теореме 1. Тогда $\lambda:=\inf K-\inf I-\inf J>0$ в том и только в том случае, когда для $\alpha=1$ или $\alpha=-1$ мы имеем

$$
\left.\left(f+\alpha S_{\pi} f\right)\right|_{(\sup I-\pi, \inf I+\lambda)}=0,\left.\quad\left(g-\alpha S_{\pi} g\right)\right|_{(\sup J-\pi, \inf J+\lambda)}=0 .
$$

Доказательство. Утверждение «только в том случае» следует из теоремы 1. Проверим утверждение «в том случае» непосредственным вычислением. Пусть $f \in \mathscr{E}^{\prime}\left(I \cup S_{\pi} I\right)$, где $I \subset \mathbb{T},|I|<\pi / 2, g \in \mathscr{E}^{\prime}\left(J \cup S_{\pi} J\right)$, где $J \subset \mathbb{T},|J|<$ $\pi / 2$, и предположим, что $f= \pm S_{\pi} f$ на $(\sup I-\pi, \inf I+\lambda), g=\mp S_{\pi} g$ на $(\sup J-\pi, \inf J+\lambda)$. Тогда, как и в $(2)$,

$$
\begin{aligned}
\left.(f * g)\right|_{(\sup I+\sup J-2 \pi, \inf I+\inf J+\lambda)}= & \left.\left.f\right|_{(\sup I-\pi, \inf I+\lambda)} * g\right|_{(\sup J-\pi, \inf J+\lambda)} \\
& +\left.\left.\left(S_{\pi} f\right)\right|_{(\sup I-\pi, \inf I+\lambda)} *\left(S_{\pi} g\right)\right|_{(\sup J-\pi, \inf J+\lambda)} \\
= & \left.\left.f\right|_{(\sup I-\pi, \inf I+\lambda)} * g\right|_{(\sup J-\pi, \inf J+\lambda)} \\
& -\left.\left.f\right|_{(\sup I-\pi, \inf I+\lambda)} * g\right|_{(\sup J-\pi, \inf J+\lambda)}=0 . \quad \square
\end{aligned}
$$

Положим $f^{\sharp}(\omega)=f(-\omega)$. Пусть $f \in \mathscr{E}^{\prime}(\mathbb{T})$, и пусть $I \subset \mathbb{T}-$ замкнутый интервал, такой, что supp $f \subset \mathscr{R}_{2}(I)$. Предположим, что не существует замкнутого интервала $I^{\prime} \subsetneq I$, такого, что $\operatorname{supp} f \subset \mathscr{R}_{2}\left(I^{\prime}\right)$. 
Теорема 2. Если $I \subset(-\pi / 2, \pi / 2)$ u $|I|<\pi / 2$, то включение $\operatorname{supp} f * f^{\sharp} \subset$ $\{0 ; \pi\}$ возможно лишь в том случае, когда $\operatorname{supp} f \subset\{\inf I ; \sup I ; \pi+\inf I ; \pi+$ $\sup I\}$. Более того, найдутся распределения $\mu, \nu \in \mathscr{E}^{\prime}(\mathbb{T})$, каждое с носителем в точке, такие, что

$$
f=\mu+S_{\pi} \mu+\nu-S_{\pi} \nu
$$

Замечание 3. Утверждение теоремы 2 останется справедливым, если мы положим $f^{\sharp}(\omega)=\overline{f(-\omega)}$. Доказательство при этом не изменится.

Наконец, приведем теорему о свертке для степеней распределения. Пусть $f \in \mathscr{E}^{\prime}(\mathbb{T})$. Пусть $I \subset \mathbb{T}-$ замкнутый интервал, такой, что $\operatorname{supp} f \subset \mathscr{R}_{n}(I)$, и предположим что не найдется интервала $I^{\prime} \subsetneq I$, такого, что $\operatorname{supp} f \subset \mathscr{R}_{n}\left(I^{\prime}\right)$.

Теорема 3 (теорема Титчмарша для степеней свертки на окружности). Пусть $|I|<2 \pi /$ рп для некоторого $p \in \mathbb{N}$. Тогда самый маленький замкнутый интервал $K \subset p I$, удовлетворяющий включению $\operatorname{supp} f^{* p} \subset \mathscr{R}_{n}(K)$, совпадает c $p I$, m.e. $K=p I$.

Мы использовали обозначения $p I=\underbrace{I+\cdots+I}_{p}$ и $f^{* p}=\underbrace{f * \cdots * f}_{p}$.

3. Доказательства. Сначала докажем следующую лемму.

Лемма 1. Пусть $f_{j} \in \mathscr{E}^{\prime}(I), j \in \mathbb{Z}_{n}$. Найдется $\alpha \in \mathbb{C}, \alpha^{n}=1$, такое, что

$$
\inf \operatorname{supp} \sum_{j \in \mathbb{Z}_{n}} \alpha^{j} f_{j}=\min _{j \in \mathbb{Z}_{n}} \inf \operatorname{supp} f_{j} .
$$

Доказательство. Положим $a:=\min _{j \in \mathbb{Z}_{n}} \inf \operatorname{supp} f_{j}$. Предположим, что, вопреки утверждению леммы, найдется $\epsilon>0$, такое, что $\inf \operatorname{supp} \sum_{j \in \mathbb{Z}_{n}} \alpha^{j} f_{j} \geqslant$ $a+\epsilon$ для любого $\alpha=\gamma^{m}$, где $\gamma=\exp (2 \pi i / n)$ и $m \in \mathbb{N}, 1 \leqslant m \leqslant n$. Тогда для любых пробных функций $\varphi \in \mathscr{D}(\mathbb{R})$ с $\operatorname{supp} \varphi \subset(a-\epsilon, a+\epsilon)$ мы имели бы

$$
0=\left\langle\varphi, \sum_{j \in \mathbb{Z}_{n}} \gamma^{j m} f_{j}\right\rangle=\sum_{j \in \mathbb{Z}_{n}} \gamma^{j m}\left\langle\varphi, f_{j}\right\rangle, \quad 1 \leqslant m \leqslant n .
$$

Пользуясь формулой для детерминанта Вандермонда, имеем

$$
\operatorname{det}\left[\begin{array}{ccccc}
1 & \gamma & \gamma^{2} & \ldots & \gamma^{n-1} \\
1 & \gamma^{2} & \gamma^{4} & \ldots & \gamma^{2(n-1)} \\
1 & \gamma^{3} & \gamma^{6} & \ldots & \gamma^{3(n-1)} \\
\vdots & \vdots & \vdots & \ddots & \vdots \\
1 & \gamma^{n} & \gamma^{2 n} & \cdots & \gamma^{n(n-1)}
\end{array}\right]=\prod_{1 \leqslant j<k \leqslant n}\left(\gamma^{k}-\gamma^{j}\right) \neq 0 .
$$

Таким образом, (11) приводит к равенству $\left\langle\varphi, f_{j}\right\rangle=0$ для всех $j \in \mathbb{Z}_{n}$. Благодаря произвольности $\varphi$ получаем $\left.f_{j}\right|_{(a-\epsilon, a+\epsilon)}=0$ для всех $j \in \mathbb{Z}_{n}$, что приводит к противоречию с определением $a$.

Доказательство теоремы 1. Имеем supp $f \subset \mathscr{R}_{n}(I), \operatorname{supp} g \subset \mathscr{R}_{n}(J)$, $\operatorname{supp} f * g \subset \mathscr{R}_{n}(K) \subset \mathscr{R}_{n}(I+J)$. В силу ограничения (4) каждое из множеств $\mathscr{R}_{n}(I), \mathscr{R}_{n}(J)$ и $\mathscr{R}_{n}(I+J)$ состоит из $n$ непересекающихся интервалов. Пусть $j \in \mathbb{Z}_{n}$; положим $f_{j}=\left.\left(S_{2 \pi j / n} f\right)\right|_{I} \in \mathscr{E}^{\prime}(I), g_{j}=\left.\left(S_{2 \pi j / n} g\right)\right|_{J} \in \mathscr{E}^{\prime}(J)$, 
$h_{j}=\left.\left(S_{2 \pi j / n}(f * g)\right)\right|_{K} \in \mathscr{E}^{\prime}(I+J)$; тогда

$$
\begin{aligned}
h_{j} & =\left.\left(S_{2 \pi j / n}(f * g)\right)\right|_{I+J} \\
& =\left.\left.\sum_{\substack{k+l \equiv j \bmod n \\
k, l \in \mathbb{Z}_{n}}}\left(S_{2 \pi k / n} f\right)\right|_{I} *\left(S_{2 \pi l / n} g\right)\right|_{J}=\sum_{\substack{k+l \equiv j \bmod n \\
k, l \in \mathbb{Z}_{n}}} f_{k} * g_{l}, \quad j \in \mathbb{Z}_{n} .
\end{aligned}
$$

Пользуясь соотношением (13), для любого $\alpha \in \mathbb{C}$, такого, что $\alpha^{n}=1$, имеем

$$
\left(\sum_{k \in \mathbb{Z}_{n}} \alpha^{k} f_{k}\right) *\left(\sum_{l \in \mathbb{Z}_{n}} \alpha^{l} g_{l}\right)=\sum_{j \in \mathbb{Z}_{n}} \alpha^{j}\left[\sum_{\substack{k+l \equiv j \bmod n \\ k, l \in \mathbb{Z}_{n}}} f_{k} * g_{l}\right]=\sum_{j \in \mathbb{Z}_{n}} \alpha^{j} h_{j}
$$

Применяя к этому соотношению теорему Титчмарша о свертке (1), получаем

$$
\inf \operatorname{supp} \sum_{j \in \mathbb{Z}_{n}} \alpha^{j} f_{j}+\inf \operatorname{supp} \sum_{j \in \mathbb{Z}_{n}} \alpha^{j} g_{j}=\inf \operatorname{supp} \sum_{j \in \mathbb{Z}_{n}} \alpha^{j} h_{j} \geqslant \inf K \text {. }
$$

Мы приняли во внимание, что $\min _{j \in \mathbb{Z}_{n}} \inf \operatorname{supp} h_{j} \geqslant \inf K$. По лемме 1 найдется $\alpha \in \mathbb{C}, \alpha^{n}=1$, такое, что $\inf \operatorname{supp} \sum_{j \in \mathbb{Z}_{n}} \alpha^{j} g_{j}=\min _{j \in \mathbb{Z}_{n}} \inf \operatorname{supp} g_{j}=\inf J$; получаем второе соотношение в (5). Для этого же значения $\alpha$ соотношение (15) дает

$$
\inf \operatorname{supp} \sum_{j \in \mathbb{Z}_{n}} \alpha^{j} f_{j} \geqslant \inf K-\inf J=\inf I+\lambda .
$$

Это приводит к первому соотношению в (5). Согласно замечанию 2 , доказательство закончено.

Доказательство теоремы 2. Если $I$ состоит из одной точки, $I=\{p\} \subset$ $(-\pi / 2, \pi / 2)$, то $\operatorname{supp} f=\mathscr{R}_{2}(p)=\{p ; \pi+p\}$ и (9) выполнено с

$$
\mu=\left.\frac{f+S_{\pi} f}{2}\right|_{I}, \quad \nu=\left.\frac{f-S_{\pi} f}{2}\right|_{I} .
$$

Предположим теперь, что $|I|>0$. Пусть $J=-I$ и $K=\{0\} \subset I+J$. Тогда $\operatorname{supp} f^{\sharp} \subset \mathscr{R}_{2}(J)$ и не существует $J^{\prime} \subsetneq J$, такого, что supp $f^{\sharp} \subset \mathscr{R}_{2}\left(J^{\prime}\right)$. Согласно условиям теоремы, $\operatorname{supp} f * f^{\sharp} \subset \mathscr{R}_{2}(K)$; таким образом,

$$
\lambda:=\inf K-\inf I-\inf J=\sup I-\inf I=|I|>0 .
$$

Применяя теорему 1 к (16), заключаем, что найдется $\alpha \in\{ \pm 1\}$, такое, что

$$
\left.\left(f+\alpha S_{\pi} f\right)\right|_{(\sup I-\pi, \sup I)}=0,
$$

а также $\left.\inf \operatorname{supp}\left(f^{\sharp}+\alpha S_{\pi} f^{\sharp}\right)\right|_{(-\pi / 2, \pi / 2)}=-\sup I ;$ последнее соотношение приводит к равенству

$$
\left.\sup \operatorname{supp}\left(f+\alpha S_{\pi} f\right)\right|_{(-\pi / 2, \pi / 2)}=\sup I .
$$

Аналогично, по теореме 1 существует $\beta \in\{ \pm 1\}$, такое, что

$$
\left.\left(f^{\sharp}+\beta S_{\pi} f^{\sharp}\right)\right|_{(-\inf I-\pi,-\inf I)}=0 ;
$$

следовательно,

$$
\left.\left(f+\beta S_{\pi} f\right)\right|_{(\inf I, \inf I+\pi)}=0 .
$$


Сравнивая (18) с (19), заключаем, что $\alpha \neq \beta$, а значит, $\alpha=-\beta$; тогда (17) и (19) позволяют заключить что и $f$, и $S_{\pi} f$ обращаются в нуль на $(\inf I, \sup I)$; следовательно,

$$
\operatorname{supp} f \subset\{\inf I ; \sup I ; \pi+\inf I ; \pi+\sup I\} \text {. }
$$

Согласно (17) и (19), если $\alpha=1$, то соотношение (9) выполняется с $\mu=$ $\left.f\right|_{(\inf I, \pi / 2)}$ и $\nu=\left.f\right|_{(-\pi / 2, \sup I)}$. Если же $\alpha=-1$, то соотношение (9) выполняется с $\mu=\left.f\right|_{(-\pi / 2, \sup I)}$ и $\nu=\left.f\right|_{(\inf I, \pi / 2)}$.

Заметим, что доказательство теоремы 3 для случая $p=2$ немедленно получается из теоремы 1. (Например, соотношения (5) с $f=g$ не приводят к противоречию, только если $\lambda=0$.) По индукции получаем доказательство для $p=2^{N}$ с любым $N \in \mathbb{N}$. Отсюда можно вывести утверждение теоремы 3 для любого $p \leqslant 2^{N}$, но при условии $|I|<2 \pi /\left(2^{N} n\right)$, которое сильнее условия $|I|<2 \pi /(p n)$. Вместо того чтобы воспользоваться теоремой 1 , дадим независимое доказательство.

Доказательство теоремы 3. Имеем supp $f^{* p} \subset \mathscr{R}_{n}(p I)$. Благодаря малости интервала $I$, и $\mathscr{R}_{n}(I)$, и $\mathscr{R}_{n}(p I)$ - наборы из $n$ непересекающихся интервалов. Положим $f_{j}:=\left.\left(S_{2 \pi j / n} f\right)\right|_{I} \in \mathscr{E}^{\prime}(I)$ и $h_{j}:=\left.\left(S_{2 \pi j / n}\left(f^{* p}\right)\right)\right|_{I} \in \mathscr{E}^{\prime}(I)$. Тогда

$$
\begin{aligned}
h_{j}=\left.\left(S_{2 \pi j / n}\left(f^{* p}\right)\right)\right|_{p I} & =\left.\left.\sum_{\substack{j_{1}+\ldots+j_{p} \equiv j \bmod n \\
j_{1}, \ldots, j_{p} \in \mathbb{Z}_{n}}}\left(S_{2 \pi j_{1} / n} f\right)\right|_{I} * \cdots *\left(S_{2 \pi j_{p} / n} f\right)\right|_{I} \\
& =\sum_{\substack{j_{1}+\cdots+j_{p} \equiv j \bmod n \\
j_{1}, \ldots, j_{p} \in \mathbb{Z}_{n}}} f_{j_{1}} * \cdots * f_{j_{p}}, \quad j \in \mathbb{Z}_{n} .
\end{aligned}
$$

Принимая во внимание (20), для любого $\alpha \in \mathbb{C}, \alpha^{n}=1$, получаем

$$
\left(\sum_{j \in \mathbb{Z}_{n}} \alpha^{j} f_{j}\right)^{* p}=\sum_{j \in \mathbb{Z}_{n}} \alpha^{j}\left[\sum_{j_{1}+\cdots+j_{p}=j \bmod n} f_{j_{1}} * \cdots * f_{j_{n}}\right]=\sum_{j \in \mathbb{Z}_{n}} \alpha^{j} h_{j} .
$$

Теперь применим к (21) теорему Титчмарша о свертке, что приводит к

$$
p \inf \operatorname{supp} \sum_{j \in \mathbb{Z}_{n}} \alpha^{j} f_{j}=\inf \operatorname{supp} \sum_{j \in \mathbb{Z}_{n}} \alpha^{j} h_{j} .
$$

По лемме 1 найдется $\alpha \in \mathbb{C}, \alpha^{n}=1$, такое, что

$$
\inf \operatorname{supp} \sum_{j \in \mathbb{Z}_{n}} \alpha^{j} f_{j}=\min _{j \in \mathbb{Z}_{n}} \inf \operatorname{supp} f_{j}
$$

следовательно, для такого $\alpha$

$$
p \min _{j \in \mathbb{Z}_{n}} \inf \operatorname{supp} f_{j}=\inf \operatorname{supp} \sum_{j \in \mathbb{Z}_{n}} \alpha^{j} h_{j} \geqslant \min _{j \in \mathbb{Z}_{n}} \inf \operatorname{supp} h_{j} .
$$

С другой стороны, (20) незамедлительно приводит к неравенствам inf $\operatorname{supp} h_{j} \geqslant$ $p \min _{k \in \mathbb{Z}_{n}} \inf \operatorname{supp} f_{k}$ для любого $j \in \mathbb{Z}_{n}$. Следовательно, $\min _{j \in \mathbb{Z}_{n}} \inf \operatorname{supp} h_{j}=$ $p \min _{j \in \mathbb{Z}_{n}} \inf \operatorname{supp} f_{j}$ и, аналогично, $\max _{j \in \mathbb{Z}_{n}} \sup \operatorname{supp} h_{j}=p \max _{j \in \mathbb{Z}_{n}} \sup \operatorname{supp} f_{j}$. 


\section{ЛитерАТУРА}

[1] Л. Хёрмандер, Анализ линейных дифберенииалъных операторов с частными производными, m. 1, Теория распределений и анализ Фуръе, Мир, М., 1986.

[2] A. I. Komech, A. A. Komech, Global attractor for a nonlinear oscillator coupled to the Klein-Gordon field, Arch. Ration. Mech. Anal., 185:1 (2007), 105-142.

[3] B. Y. Levin, Lectures on Entire Functions. In collaboration with Yu. Lyubarskii, M. Sodin and V. Tkachenko, Transl. Math. Monographs, vol. 150, Amer. Math. Soc., Providence, RI, 1996.

[4] J.-L. Lions, Supports de produits de composition. I, C. R. Acad. Sci. Paris, 232 (1951), 1530-1532.

[5] W. Strauss, L. Vazquez, Numerical solution of a nonlinear Klein-Gordon equation, J. Comput. Phys., 28:2 (1978), 271-278.

[6] E. Titchmarsh, The zeros of certain integral functions, Proc. London Math. Soc., 25 (1926), 283-302.

[7] К. Иосида, Функциональный анализ, Мир, М., 1967.

Техасский университет сельского хозяйства и механизации Институт проблем передачи информации e-mail: comech@math.tamu.edu

Венский университет

Институт проблем передачи информации

e-mail: alexander.komech@univie.ac.at
Поступило в редакцию 9 января 2011 г. 